\title{
Positron range in PET imaging: non-conventional isotopes
}

\section{Running head: Positron range for non-conventional isotopes}

\author{
Authors \\ L. Jødal ${ }^{1}$, C. Le Loirec ${ }^{2}$, C. Champion ${ }^{3}$ \\ ${ }^{1}$ Department of Nuclear Medicine, Aalborg University Hospital (Aalborg, Denmark) \\ ${ }^{2}$ CEA, LIST, F-91191 Gif-sur-Yvette, France (Gif-sur-Yvette, France) \\ ${ }^{3}$ Centre d'Études Nucléaires de Bordeaux Gradignan, CNRS/IN2P3, Université Bordeaux \\ (Gradignan, France)
}

\begin{abstract}
Background: In addition to conventional short-lived radionuclides, longer-lived isotopes are becoming increasingly important to PET. The longer half-life both allows for circumvention of the in-house production of radionuclides, and expands the spectrum of physiological processes amenable to PET imaging, including processes with prohibitively slow kinetics for investigation with short-lived radiotracers. However, many of these radionuclides emit "high-energy" positrons and gamma rays which affect the spatial resolution and quantitative accuracy of PET images.

Aims: The objective of the present work is to investigate the positron range distribution for some of these long lived isotopes.

Methods: Based on existing Monte Carlo simulations of positron interactions in water, the probability distribution of the line of response displacement have been empirically described by means of analytic displacement functions.

Results: Relevant distributions have been derived for the isotopes ${ }^{22} \mathrm{Na},{ }^{52} \mathrm{Mn},{ }^{89} \mathrm{Zr},{ }^{45} \mathrm{Ti},{ }^{51} \mathrm{Mn},{ }^{94 \mathrm{~m}} \mathrm{Tc}$, ${ }^{52 \mathrm{~m}} \mathrm{Mn},{ }^{38} \mathrm{~K},{ }^{64} \mathrm{Cu},{ }^{86} \mathrm{Y},{ }^{124} \mathrm{I}$, and ${ }^{120} \mathrm{I}$. It was found that the distribution functions previously found for a series of conventional isotopes (Jødal et al, Phys Med Bio 57:3931-3943), were also applicable to these
\end{abstract}


non-conventional isotopes, except that for ${ }^{120} \mathrm{I},{ }^{124} \mathrm{I},{ }^{89} \mathrm{Zr},{ }^{52} \mathrm{Mn}$, and ${ }^{64} \mathrm{Cu}$, parameters in the formulae were less well predicted by mean positron energy alone.

Conclusions: Both conventional and non-conventional range distributions can be described by relatively simple analytic expressions. The results will be applicable to image-reconstruction software to improve the resolution.

Keywords: Monte Carlo simulation, positron range, spatial resolution, annihilation, PET, point spread function

PACS classification: 87.57.uk (Positron emission tomography)

Submitted to: Physics in Medicine and Biology 


\section{Introduction}

One of the greatest advantages of positron emission tomography (PET) is the use of "bio-isotopes" such as ${ }^{11} \mathrm{C},{ }^{13} \mathrm{~N}$ and ${ }^{15} \mathrm{O}$ for the investigation of biological processes. These three isotopes are shortlived and decay into non-radioactive daughters. They can thus be administered in large quantities without exposing the patient to high doses. However, these bio-isotopes suffer from a major drawback because of their very short half-lives: they cannot be transported over long distances and, therefore, access to cyclotron and radiochemistry facilities is necessary for the on-demand preparation of the desired radiotracers (Pagani et al. 1997).

From this point of view, the use of long-lived radionuclides would circumvent the complexities associated with the in-house production of radionuclides. Additionally, it is worth noting that the investigable spectrum of physiological processes would expand if more types of isotopes could be employed.

The study of slow biochemical processes requires access to radionuclides with half-lives of many hours. For example, the use of positron-emitter-labeled monoclonal antibodies that combine the specificity of an antibody with the resolution of PET requires radionuclides with half-lives that match the half-lives of the antibodies in the circulatory system (i.e., $>48$ hours). In this context, ${ }^{124} \mathrm{I}$ and ${ }^{89} \mathrm{Zr}$ - with half-lives of 100 and 78 hours, respectively - are potentially suitable for this purpose (Disselhorst et al. 2010). Moreover, recent advances in radioimmunotherapy have demonstrated the benefit of using long-lived radionuclides for diagnosis and therapy. Indeed, the use of ligands with high affinity with long-lived radionuclides allows for the analysis of the "wash-out" phases. As an example, ${ }^{64} \mathrm{Cu}$-labeled antibodies can be used to estimate the dosimetry prior to ${ }^{67} \mathrm{Cu}$ therapy (Pagani et al. 1997). The half-life of ${ }^{124}$ I is also well suited to in vivo studies of the prolonged time course of the uptake of monoclonal antibodies in solid tumors (Daghighian et al. 1993; Larson et al. 1992; Snyder et al. 1975). Because of its long half-life, which allows sequential acquisitions to be conducted 
over several days, ${ }^{86} \mathrm{Y}\left(\mathrm{T}_{1 / 2}=14.7 \mathrm{~h}\right)$ can also be used to quantitatively determine the pharmacokinetics of ${ }^{90} \mathrm{Y}$, which is commonly used in the palliative treatment of bone metastases (Herzog et al. 1993; Rösch et al. 1996).

The investigation of cationic fluxes requires a cationic tracer such as ${ }^{38} \mathrm{~K}^{+}$, which is used to probe myocardial perfusion (Bol et al. 1993; De Landsheere et al. 1992). Moreover, ${ }^{51} \mathrm{Mn}$ and ${ }^{52} \mathrm{Mn}$ can also be used as cationic perfusion tracers (Daube and Nickles 1985) and have also been proposed for the diagnosis and treatment of blood disease (Sastri et al. 1981).

However, to be useful, a PET isotope not only must be applicable to a relevant physiological measure but also must be considered in terms of its physical aspects of positron emission with respect to image quality and/or accurate quantification. One such aspect is emission of high-energy ( $\geq 500$ $\mathrm{keV}$ ) gamma rays, not originating from positron annihilations, but which may still be detected within the $511 \mathrm{keV}$ energy window. Neither of the isotopes ${ }^{11} \mathrm{C},{ }^{13} \mathrm{~N}$ and ${ }^{15} \mathrm{O}$ emit high-energy gamma rays. A more fundamental aspect is positron range: The PET scanner measures photons emitted from the position of positron annihilation, which will generally differ somewhat from the position of positron emission (and thus differ from the position of the PET isotope). Higher energy of the emitted positrons will lead to higher positron ranges.

Indeed, some of the non-conventional isotopes mentioned above emit high-energy positrons and gamma rays, which affect the spatial resolution of PET imaging and increase the randoms contribution (Laforest et al. 2002). Several groups have thus reported on high-resolution (including small-animal) PET to determine the impact of non-conventional isotopes on the image quality. Laforest (2002), Liu and Laforest (2009), and Disselhorst et al. (2010) have demonstrated that the positron range affects both the spatial resolution and the PET image contrast. Furthermore, they have noted that the long-range tail of the positron-range distribution considerably diminishes the contrast of the image but that the end-point of the energy of the $\beta$ spectrum alone is insufficient to characterize 
the potential image quality of a given PET isotope. De Jong et al. (2005) and Kemerink et al. (2011) have concluded that none of the imaging characteristics of such non-conventional isotopes prevent the use of these isotopes in high-resolution quantitative PET imaging.

If the distribution of positron ranges is known, however, it is possible to include this information in the image reconstruction and thereby reduce the effect of positron range. Two classical papers on the subject are (Derenzo 1986) and (Levin and Hoffmann 1999). In a previous study (Jødal et al. 2012) we described mathematical distributions of positron range for a series of commonly used PET isotopes.

The objective of the present study is to investigate the positron-range distribution for the following "non-conventional", but promising, PET isotopes: ${ }^{22} \mathrm{Na},{ }^{52} \mathrm{Mn},{ }^{89} \mathrm{Zr},{ }^{45} \mathrm{Ti},{ }^{51} \mathrm{Mn},{ }^{94 \mathrm{~m}} \mathrm{Tc}$, ${ }^{52 \mathrm{~m}} \mathrm{Mn}$, and ${ }^{38} \mathrm{~K}$ (all of the allowed-decay type - see section 2 ); ${ }^{64} \mathrm{Cu}$ (of the allowed-decay type, but emitting both $\beta^{+}$and $\beta$ ); and ${ }^{124} \mathrm{I},{ }^{86} \mathrm{Y}$, and ${ }^{120} \mathrm{I}$ (of the forbidden [non-allowed] decay type). For these isotopes, we will use the same framework that was developed in our previous study (Jødal et al. 2012), in which we argued that a 2D projection of the 3D positron-range distribution was convenient for modeling because an LOR is a $2 \mathrm{D}$ measurement rather than a $3 \mathrm{D}$ one (see Figure 1). The probability distributions of the line of response (LOR) displacement $\delta$ will be sought and empirically described by means of analytic functions.

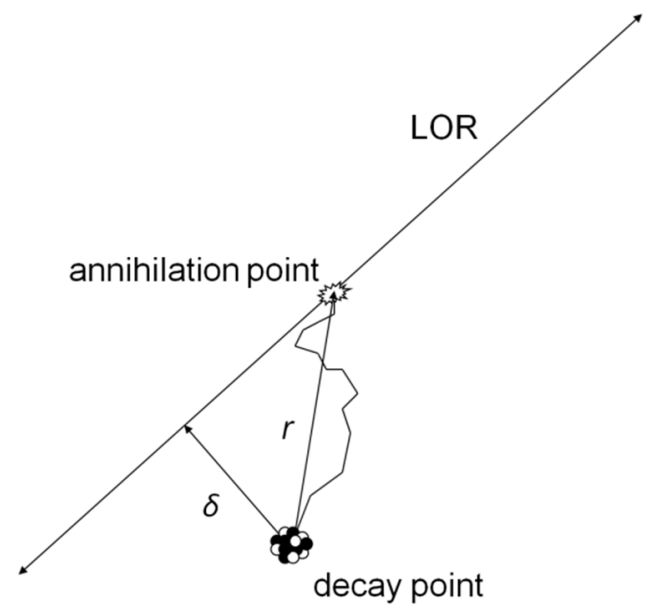


Figure 1: The positron moves in 3 dimensions with range $r$ being the distance to the annihilation point. However, the PET scanner does not measure the position of the annihilation point, only the position of the line defined by the detected photons (the line of response, LOR), thus reducing the number of dimensions to 2. Even for Time-of-Flight (TOF) PET, a line of several centimeters in length is measured, not a point. This means that the LOR displacement $(\delta)$, rather than the positron range $(r)$, is the quantity that is directly related to the measured data.

\section{Methods common to all studied isotopes}

The system spatial resolution of PET is limited by a number of factors, including the positron range. However, if accurate range distributions can be incorporated into the image-reconstruction process, improved resolution may be achieved (Alessio and MacDonald 2008; Bai et al. 2003).

For this purpose, we have recently proposed a first study focused on commonly used PET isotopes. The 2D range distributions of ${ }^{18} \mathrm{~F},{ }^{11} \mathrm{C},{ }^{13} \mathrm{~N},{ }^{15} \mathrm{O},{ }^{68} \mathrm{Ga},{ }^{62} \mathrm{Cu}$, and ${ }^{82} \mathrm{Rb}$ (Jødal et al. 2012) were analytically characterized by a set of parameters expressed from the mean positron energy $E_{\text {mean }}$. However, this initial study was limited to isotopes of the allowed-decay type, that is, with a decay in which parity remains unchanged and the nuclear spin changes by 0 or \pm 1 (Krane 1988). Physically, "allowed" decays are decays in which no orbital momentum is transferred to the emitted $\beta$ particle. Mathematically, this corresponds to the lowest-order term in the transition matrix, and for such transitions, it is possible to give analytical formulae for the beta energy spectrum (Krane 1988; Venkataramaiah et al. 1985). Thus, the allowed-decay isotopes share certain common features, which may be the reason for our success using a simple description.

When possible, the allowed decays of a nuclide usually have the highest transition probabilities, but if the daughter nucleus possesses only quantum states with different parity and/or with nuclear spin differing by 2 or more from the parent state, a forbidden (sometimes also called "non-allowed") 
transition is the only option. These transitions involve an exchange of orbital momentum between the nucleus and the $\beta$ particle, and the resulting energy spectra are more varied than those for allowed decays.

Many of the longer-lived PET isotopes are of the forbidden-decay type, for example, the isotope ${ }^{124} \mathrm{I}$, which undergoes $\beta^{+}$decay of types $2^{-} \rightarrow 0^{+}$and $2^{-} \rightarrow 2^{+}$(Lederer et al. 1967). In PET, ${ }^{124} \mathrm{I}$ is used to scan slow physiological processes over a period of several days, such as in the in vivo study of the uptake of monoclonal antibodies in solid tumors (Daghighian et al. 1993; Larson et al. 1992; Snyder et al. 1975). Another example of a clinically relevant forbidden-decay PET isotope is ${ }^{86} \mathrm{Y}$, which is used for PET scanning prior to therapy using ${ }^{90} \mathrm{Y}$ (Herzog et al. 1993; Rösch et al. 1996). One-third of the decays of ${ }^{86} \mathrm{Y}$ lead to the emission of positrons with maximum energies ranging from $2.34 \mathrm{MeV}$ ( $4.6 \%$ of the positron-emitting decays) to $1.04 \mathrm{MeV}$ (15\% of the positron-emitting decays) (Pagani et al. 1997). The isotope ${ }^{120} \mathrm{I}$ has also been suggested for use in PET applications (Herzog et al. 2006). This isotope is also of the forbidden-decay type. It has been proposed as an alternative to ${ }^{124}$ I for applications in which a shorter half-life and higher positron emission are required (Zweit et al. 1996).

Another long-lived PET isotope is ${ }^{64} \mathrm{Cu}$, which is of the allowed-decay type $\left(1^{+} \rightarrow 0^{+}\right)$but exhibits both $\beta^{-}$and $\beta^{+}$decay. Including the electron capture (EC) decay, its disintegration pathways are as follows:

$$
\begin{array}{ll}
\beta^{-}(38 \%): & { }_{29}^{64} \mathrm{Cu} \rightarrow{ }_{30}^{64} \mathrm{Zn}+{ }_{-1}^{0} \mathrm{e}+\bar{v} \\
\beta^{+}(19 \%): & { }_{29}^{64} \mathrm{Cu} \rightarrow{ }_{28}^{64} \mathrm{Ni}+{ }_{+1}^{0} \mathrm{e}+v \\
\mathrm{EC}(43 \%): & { }_{29}^{64} \mathrm{Cu}+{ }_{-1}^{0} \mathrm{e} \rightarrow{ }_{28}^{64} \mathrm{Ni}+v
\end{array}
$$

Under these conditions, it is not obvious that this particular isotope will exhibit the same behavior as previously studied allowed-decay isotopes, which undergo only $\beta^{+}$(and EC) decay. 
The list of investigated allowed-decay isotopes was extended to other promising PET isotopes (see Table 1) to confirm the ability of our proposed method to provide a uniform approach to the determination of $2 \mathrm{D}$ range distributions.

Table 1: Studied isotopes

\begin{tabular}{|c|c|c|c|c|c|c|c|}
\hline Isotope & $\begin{array}{c}\text { Allowed } \\
\text { decay }\end{array}$ & $\mathbf{T}_{1 / 2}$ & $\begin{array}{l}\text { Emean } \\
(\mathrm{keV})\end{array}$ & $\begin{array}{c}\text { Emax } \\
(\mathrm{keV})\end{array}$ & $\begin{array}{c}R_{\text {mean }} \\
(\mathbf{m m})^{\mathbf{a}, \mathbf{b}}\end{array}$ & $\begin{array}{c}\mathbf{R}_{\max } \\
(\mathbf{m m})^{\mathbf{a}, \mathbf{b}}\end{array}$ & Example of application \\
\hline${ }^{64} \mathrm{Cu}$ & $\begin{array}{l}\text { Yes, } \beta^{+} \\
\text {and } \beta^{-}\end{array}$ & $12.7 \mathrm{~h}$ & 216 & 653.1 & 0.56 & 2.9 & $\begin{array}{c}\text { Detection of small } \\
\text { colorectal tumors } \\
\text { (Philpott et al. } 1995 \text { ) }\end{array}$ \\
\hline${ }^{22} \mathrm{Na}$ & $\mathrm{Yes}^{\mathrm{c}}$ & $2.6 \mathrm{y}$ & 220.3 & 1820.2 & $0.53^{\mathrm{d}}$ & $2.28^{d}$ & Scanner calibration \\
\hline${ }^{52} \mathrm{Mn}$ & Yes & $5.6 \mathrm{~d}$ & 244.6 & 575.8 & 0.63 & 2.5 & $\begin{array}{l}\text { Candidate for bone scanning } \\
\text { (Topping et al. 2013) }\end{array}$ \\
\hline${ }^{89} \mathrm{Zr}$ & Yes & $78.4 \mathrm{~h}$ & 402.7 & 902 & 1.27 & 4.2 & $\begin{array}{l}\text { Quantifying the deposition } \\
\text { of monoclonal antibodies in } \\
\text { the tissues of tumors } \\
\text { (Link et al. 2006) }\end{array}$ \\
\hline${ }^{45} \mathrm{Ti}$ & Yes & $3.08 \mathrm{~h}$ & 442.3 & 1040.4 & 1.47 & 5.2 & $\begin{array}{l}\text { Uptake measurements to } \\
\text { provide insight into the } \\
\text { mechanism of the action of } \\
\text { titanocene dichloride } \\
\text { (Vavere and Welch 2005) }\end{array}$ \\
\hline${ }^{51} \mathrm{Mn}$ & Yes & $46.2 \mathrm{~m}$ & 970.2 & 2185.8 & 4.3 & 12.1 & $\begin{array}{c}\text { Diagnosis and treatment of } \\
\text { blood diseases } \\
\text { (Sastri et al. } 1981)\end{array}$ \\
\hline${ }^{94 m} \mathbf{T c}$ & Yes & $52.0 \mathrm{~m}$ & 1076.6 & 2362.9 & 4.7 & 12.8 & $\begin{array}{l}\text { PET substitute for }{ }^{99 m} \mathrm{Tc} \\
\text { (Liu and Laforest 2007) }\end{array}$ \\
\hline${ }^{52 \mathrm{~m}} \mathrm{Mn}$ & Yes & $21.1 \mathrm{~m}$ & 1179 & 2630 & 5.3 & 14.5 & $\begin{array}{c}\text { Myocardial imaging } \\
\text { (Daube and Nickles 1985; } \\
\text { Hui et al. } 1979 \text {; Tolmachev } \\
\text { et al. 1994) }\end{array}$ \\
\hline${ }^{38} \mathbf{K}$ & Yes & $7.64 \mathrm{~m}$ & 1218.8 & 2926 & 5.7 & 15.3 & $\begin{array}{l}\text { Myocardial perfusion } \\
\text { (Bol et al. 1993) }\end{array}$ \\
\hline${ }^{86} \mathbf{Y}$ & No & $14.7 \mathrm{~h}$ & 640 & 3141.3 & 2.5 & 11.1 & $\begin{array}{l}\text { Uptake measurements prior } \\
\text { to therapy using }{ }^{90} \mathrm{Y} \text { (Rösch } \\
\text { et al. } 1996)\end{array}$ \\
\hline${ }^{124} \mathbf{I}$ & No & $4.176 \mathrm{~d}$ & 825.9 & 2137.6 & 3.4 & 11.7 & $\begin{array}{l}\text { Uptake of monoclonal } \\
\text { antibodies in solid tumors } \\
\text { (Daghighian et al. 1993; } \\
\text { Larson et al. 1992; Snyder } \\
\text { et al. 1975) }\end{array}$ \\
\hline${ }^{120} \mathbf{I}$ & No & $81 \mathrm{~m}$ & 1747 & 4600 & 8.3 & 27 & $\begin{array}{l}\text { Shorter lifetime and higher } \\
\text { positron yield than }{ }^{124} \mathrm{I} \\
\text { (Zweit et al. } 1996)\end{array}$ \\
\hline
\end{tabular}


a (Le Loirec and Champion 2007a)

${ }^{\mathrm{b}}$ (Le Loirec and Champion 2007b)

${ }^{\mathrm{c}}$ Decay to the ground state of ${ }^{22} \mathrm{Ne}$ is forbidden $\left(3^{+} \rightarrow 0^{+}\right)$; instead, nearly $100 \%$ of the decays are to an excited state, ${ }^{22} \mathrm{Na}\left(3^{+}\right) \rightarrow{ }^{22} \mathrm{Ne}^{*}\left(2^{+}\right)$(Lederer et al. 1967)

d (Le Loirec 2007)

\section{Geometry and notation}

In a homogeneous medium and in the absence of (strong) magnetic fields, the situation is spherically symmetrical, that is, with an annihilation-point distribution per volume that may be described by a function $f_{3 \mathrm{D}}(x, y, z)=f_{3 \mathrm{D}}(r)$, where $r=\sqrt{x^{2}+y^{2}+z^{2}}$ is the range of a positron that annihilates at position $(x, y, z)$. As discussed in our previous paper (Jødal et al. 2012), such density distributions have so sharp peaks that maximum values of the distribution (whether determined through measurement or simulation) can be considerably influenced by the resolution, for which reason fullwith-at-half-maximum (FWHM) values become questionable or even meaningless. A basic reason for these sharp peaks is the $1 / r^{2}$ effect of increasing range (3D radius). Instead, we will use a notation that represents a radial distribution, which, in the $3 \mathrm{D}$ case, corresponds to the density $g_{3 \mathrm{D}}(r)$ of annihilations for a given range $r$. Geometrically, $g_{3 \mathrm{D}}(r)=4 \pi r^{2} f_{3 \mathrm{D}}(r)$.

However, as reported above and as described in detail in our previous work (Jødal et al. 2012), we consider here the 2D distribution of LOR displacements (denoted by $\delta$ ) instead of the 3D distribution of the positron range $r$ (see Figure 1). Because of the spherical symmetry, the 2D distribution can be obtained by projecting the 3D distribution onto an arbitrary plane, e.g., the $x y$ plane. In such a projection, the situation is cylindrically symmetrical, that is, the distribution of annihilations per area can be described by the function $f_{2 \mathrm{D}}(x, y)=f_{2 \mathrm{D}}(\delta)$, where $\delta=\sqrt{x^{2}+y^{2}}$ is the 
LOR displacement for a given annihilation. The radial density of displacements is given by $g_{2 \mathrm{D}}(\delta)$, where $g_{2 \mathrm{D}}(\delta)=2 \pi \delta f_{2 \mathrm{D}}(\delta)$.

The cumulative probability distribution $G_{2 \mathrm{D}}(\delta)$, which represents the probability that a given annihilation occurs with a displacement of at most $\delta$, is simply the integral of $g_{2 \mathrm{D}}(\delta)$ from 0 to $\delta$. Thus, we can write:

$$
\begin{aligned}
& \text { fraction of annihilations with a } 2 D \text { displacement of at most } \delta=G_{2 D}(\delta) \\
& \qquad=\int_{0}^{\delta} g_{2 D}\left(\delta^{\prime}\right) d \delta^{\prime} \\
& =\int_{0}^{\delta} 2 \pi \delta^{\prime} f_{2 D}\left(\delta^{\prime}\right) d \delta^{\prime} \\
& =\iint_{x^{2}+y^{2} \leq \delta^{2}} f_{2 D}(x, y) d x d y
\end{aligned}
$$

\section{Positron-energy spectra and Monte Carlo simulations}

The 3D distributions of annihilation points for positron emission in water reported in previous studies (Champion and Le Loirec 2007; Le Loirec and Champion 2007a; Le Loirec and Champion 2007b) were used. In brief, the energy spectra for the allowed-decay isotopes could be described analytically, whereas the spectra for the forbidden-decay isotopes were determined using fits to published energy spectra. Using the Monte Carlo track-structure code EPOTRAN (Champion et al. 2012), a large number ( 250000 for each isotope studied) of positron tracks were followed through liquid water (density of $1.00 \mathrm{~g} / \mathrm{cm}^{3}$ ), taking into account the formation of positronium during the slowing-down process. This simulation provided detailed data on track structure, energy deposition, and the spatial distribution of annihilation points. In the present study, only the latter was used.

The radial 2D distribution of LOR displacements $g_{2 \mathrm{D}}(\delta)$ was determined from these data by

calculating $\delta=\sqrt{x^{2}+y^{2}}$ for each annihilation event, binning the resulting values into $10 \mu \mathrm{m}$ or 300 
$\mu \mathrm{m}$ bins (depending on whether $E_{\text {mean }}$ was below or above $1000 \mathrm{keV}$, respectively), and dividing by the total number of annihilations for the given isotope.

Finally, the cumulative probability distribution $G_{2 \mathrm{D}}(\delta)$ was calculated by summing $g_{2 \mathrm{D}}(\delta)$ over all bins up to $\delta$.

\section{Distributions of the displacement $\delta$}

Analytical approximation of the $2 D$ distributions

As in our previous study (Jødal et al. 2012), we approximate the cumulative distributions as follows:

$$
G_{2 D}(\delta) \approx \zeta(\delta)=1-\exp \left(-A \delta^{2}-B \delta\right)
$$

If both $A$ and $B$ are positive numbers, then $-A \delta^{2}-B \delta$ will monotonically decrease from 0 toward $-\infty$, yielding a cumulative probability distribution with the physically correct behavior of being equal to 0 at $\delta=0$ and monotonically increasing to 1 .

Mathematically, the function has no maximal positron range, but even without a well-defined maximal range, it correctly predicts that in practice, no annihilations will be observed at large distances: as the quadratic term becomes large, the difference between 1 and $\zeta(\delta)$ will become practically 0 .

The radial density distribution will then be approximated as follows:

$$
g_{2 D}(\delta) \approx \frac{d \zeta}{d \delta}=(2 A \delta+B) \exp \left(-A \delta^{2}-B \delta\right)
$$

The parameters $A$ and $B$ are empirical parameters, but setting $\delta=0$ in Eq. (3) yields $g_{2 \mathrm{D}}(0) \approx B$, allowing us to interpret $B$ as the initial value of the radial 2D density distribution, or the initial slope of the cumulative distribution. The parameter $A$ has no physical interpretation. 


\section{Determination of $A$ and $B$}

As is generally the case when evaluating from a finite number of data points, the cumulative distribution, $G_{2 \mathrm{D}}(\delta)$, will be more robustly determined than the differential distribution, $g_{2 \mathrm{D}}(\delta)$. For this reason, the best-fit values of $A$ and $B$ were determined using GNUplot by fitting the function represented by Eq. (2) to the Monte Carlo data for $G_{2 \mathrm{D}}(\delta)$ (see Figures 2 and 3).

Since the results are to be applied as differential distributions, we also compared our results with the Monte Carlo data for $\mathrm{g}_{2 \mathrm{D}}(\delta)$, finding that a satisfactory fit was achieved for both allowed-decay and forbidden-decay isotopes, although for ${ }^{86} \mathrm{Y}$ and ${ }^{124} \mathrm{I}$, the fit was less successful for the lowest displacement values (see Figures 4 to 7). The parameter values for $A$ and $B$ are presented in Table 2 .

Table 2: Fitting parameters for the empirical cumulative probability given by (1). The standard errors are provided in brackets for each isotope.

\begin{tabular}{ccc}
\hline Isotopes & $\boldsymbol{A}\left(\mathrm{mm}^{-\mathbf{2}}\right)$ & $\boldsymbol{B}\left(\mathbf{m m}^{-\mathbf{1}}\right)$ \\
\hline${ }^{{ }^{64} \mathbf{C u}}$ & $0.0369( \pm 56.7 \%)$ & $2.0381( \pm 0.84 \%)$ \\
${ }^{{ }^{22} \mathbf{N a}}$ & $0.3391( \pm 3.7 \%)$ & $2.0645( \pm 0.4 \%)$ \\
${ }^{52} \mathbf{M n}$ & $0.6316( \pm 0.9 \%)$ & $1.3722( \pm 0.29 \%)$ \\
${ }^{{ }^{89}} \mathbf{Z r}$ & $0.2433( \pm 0.5 \%)$ & $0.5076( \pm 0.32 \%)$ \\
${ }^{45} \mathbf{T i}$ & $0.1419( \pm 0.62 \%)$ & $0.5055( \pm 0.28 \%)$ \\
${ }^{51} \mathbf{M n}$ & $0.0291( \pm 0.33 \%)$ & $0.1218( \pm 0.33 \%)$
\end{tabular}




\begin{tabular}{ccc}
\hline${ }^{{ }^{94 m} \mathbf{T c}}$ & $0.0252( \pm 1.9 \%)$ & $0.1062( \pm 2.1 \%)$ \\
${ }^{{ }^{52} \mathbf{M n}}$ & $0.0195( \pm 2.1 \%)$ & $0.0979( \pm 2.2 \%)$ \\
${ }^{38} \mathbf{K}$ & $0.018( \pm 2.2 \%)$ & $0.0936( \pm 2.2 \%)$ \\
${ }^{86} \mathbf{Y}$ & $0.0191( \pm 11.7 \%)$ & $0.4562( \pm 1.42 \%)$ \\
${ }^{{ }^{124}} \mathbf{I}$ & $0.0267( \pm 1.58 \%)$ & $0.2418( \pm 0.64 \%)$ \\
${ }^{{ }^{120}} \mathbf{I}$ & $0.0073( \pm 0.84 \%)$ & $0.0660( \pm 0.76 \%)$ \\
\hline
\end{tabular}

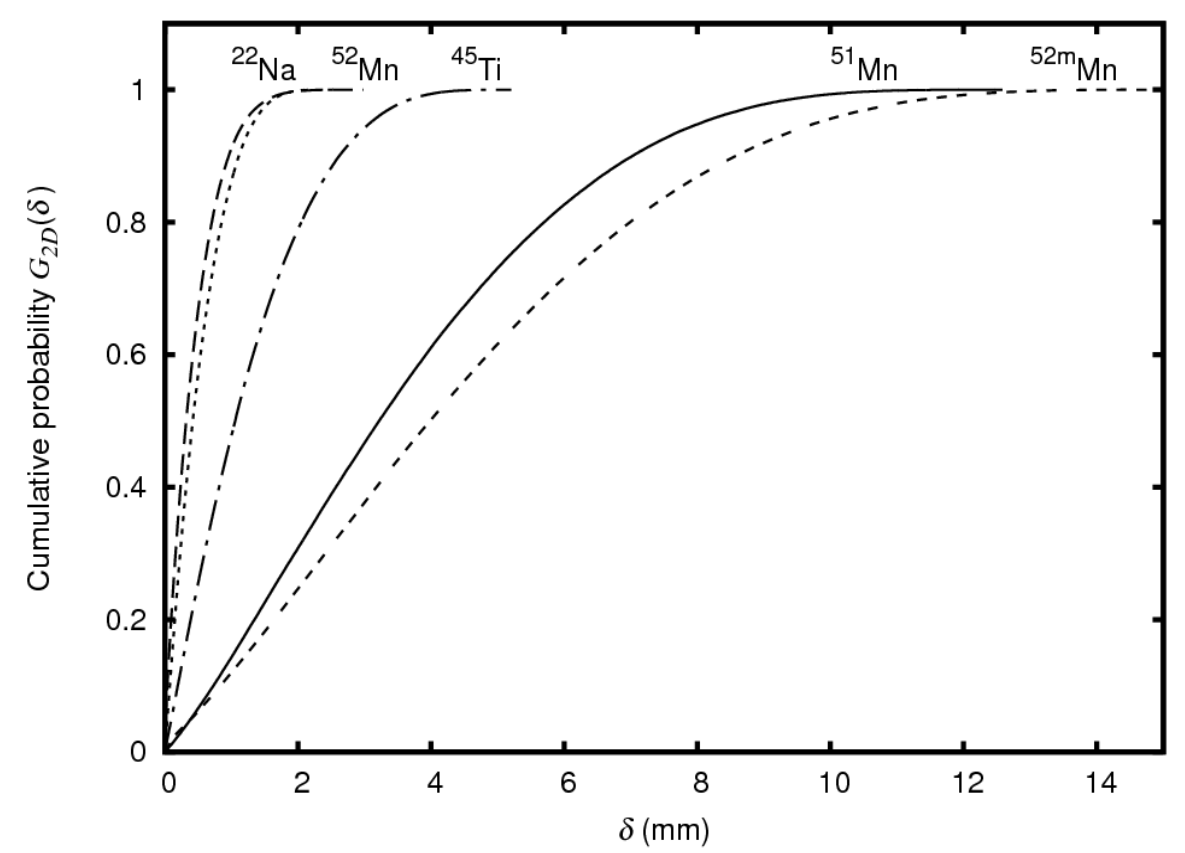

Figure 2: Cumulative probabilities $G_{2 D}(\delta)$ obtained for the following isotopes: ${ }^{22} \mathrm{Na},{ }^{52} \mathrm{Mn},{ }^{45} \mathrm{Ti},{ }^{51} \mathrm{Mn}$ and ${ }^{52 \mathrm{~m}} \mathrm{Mn}$. 


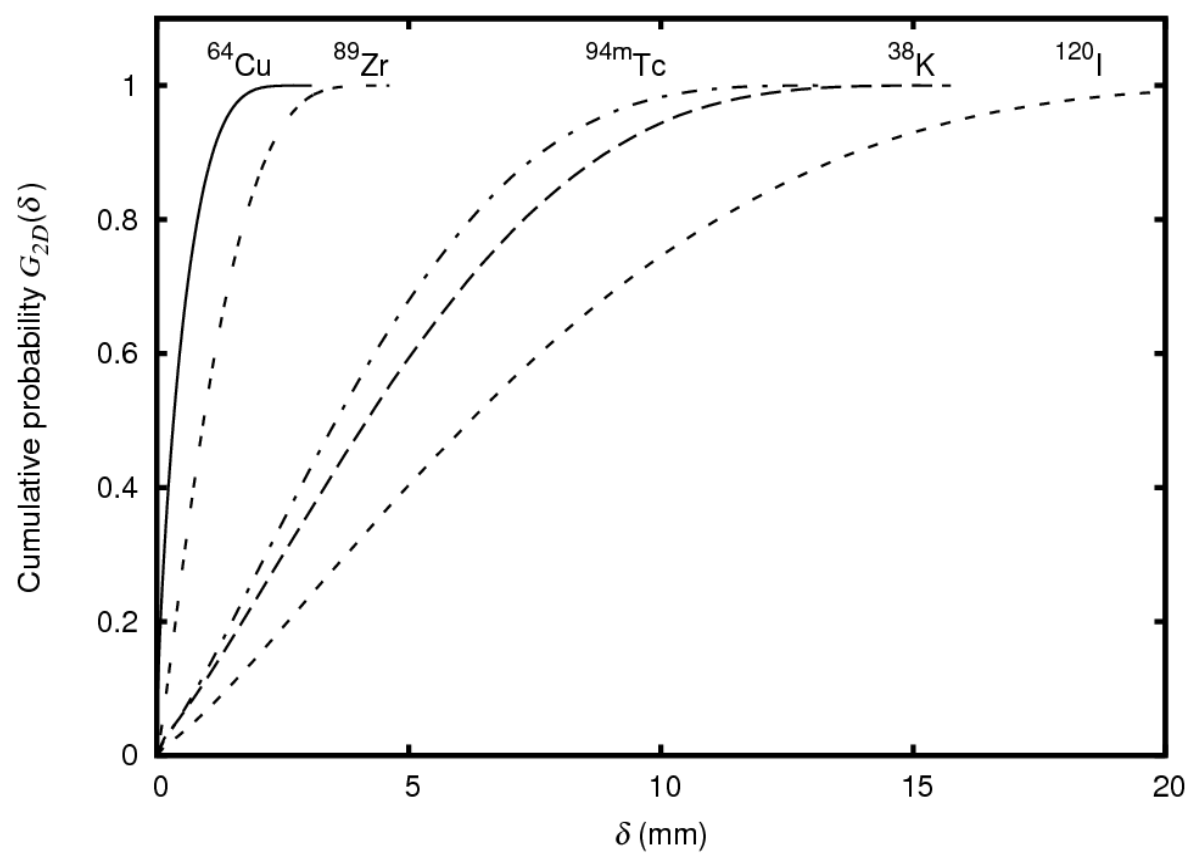

Figure 3: Cumulative probabilities $G_{2 D}(\delta)$ obtained for the following isotopes: ${ }^{64} \mathrm{Cu},{ }^{89} \mathrm{Zr},{ }^{94 \mathrm{~m}} \mathrm{Tc},{ }^{38} \mathrm{~K}$ and ${ }^{120} \mathrm{I}$.
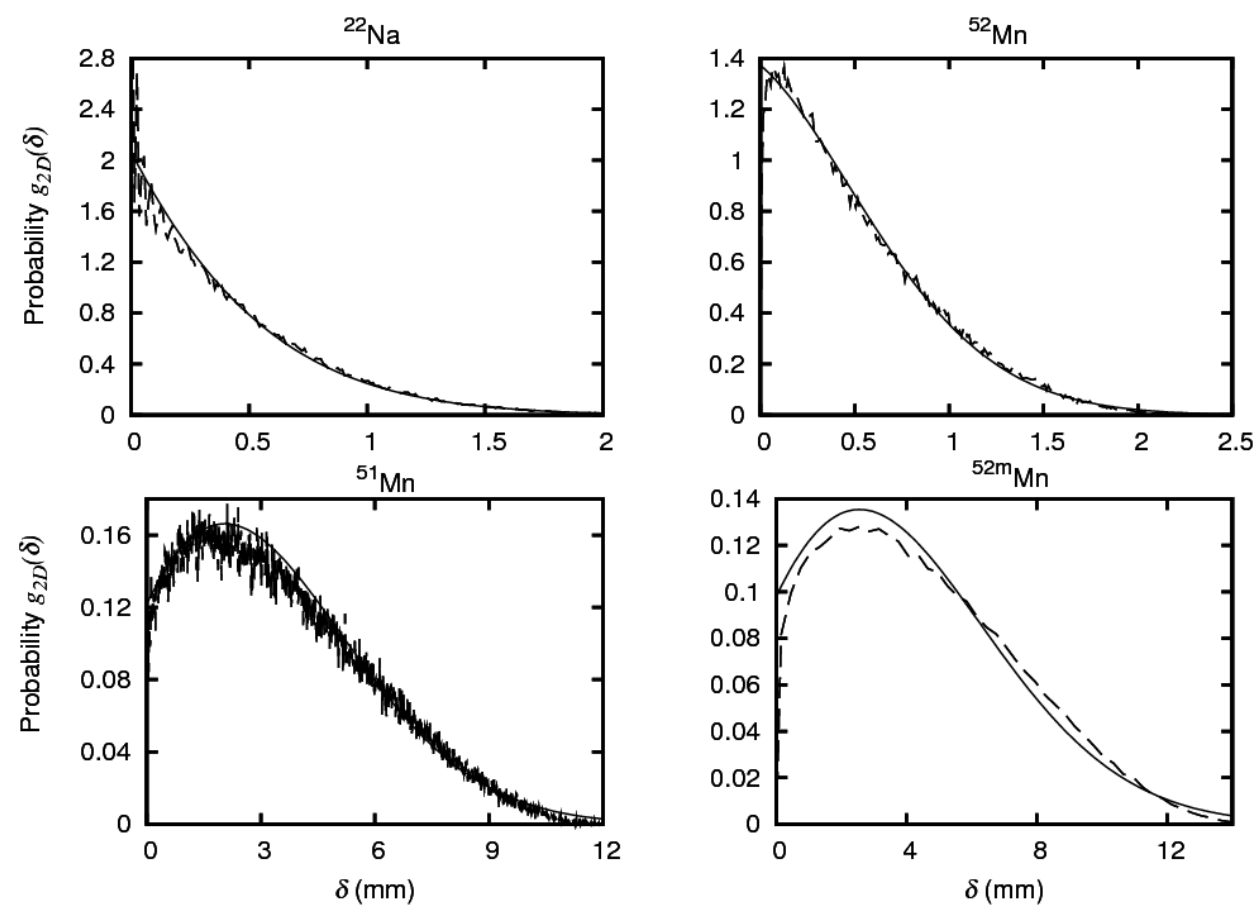

Figure 4: Functions $g_{2 D}(\delta)$ obtained from Monte Carlo calculations (broken lines) compared with the expressions defined by (3) using the values of $A$ and $B$ provided in table 2 (smooth lines). 

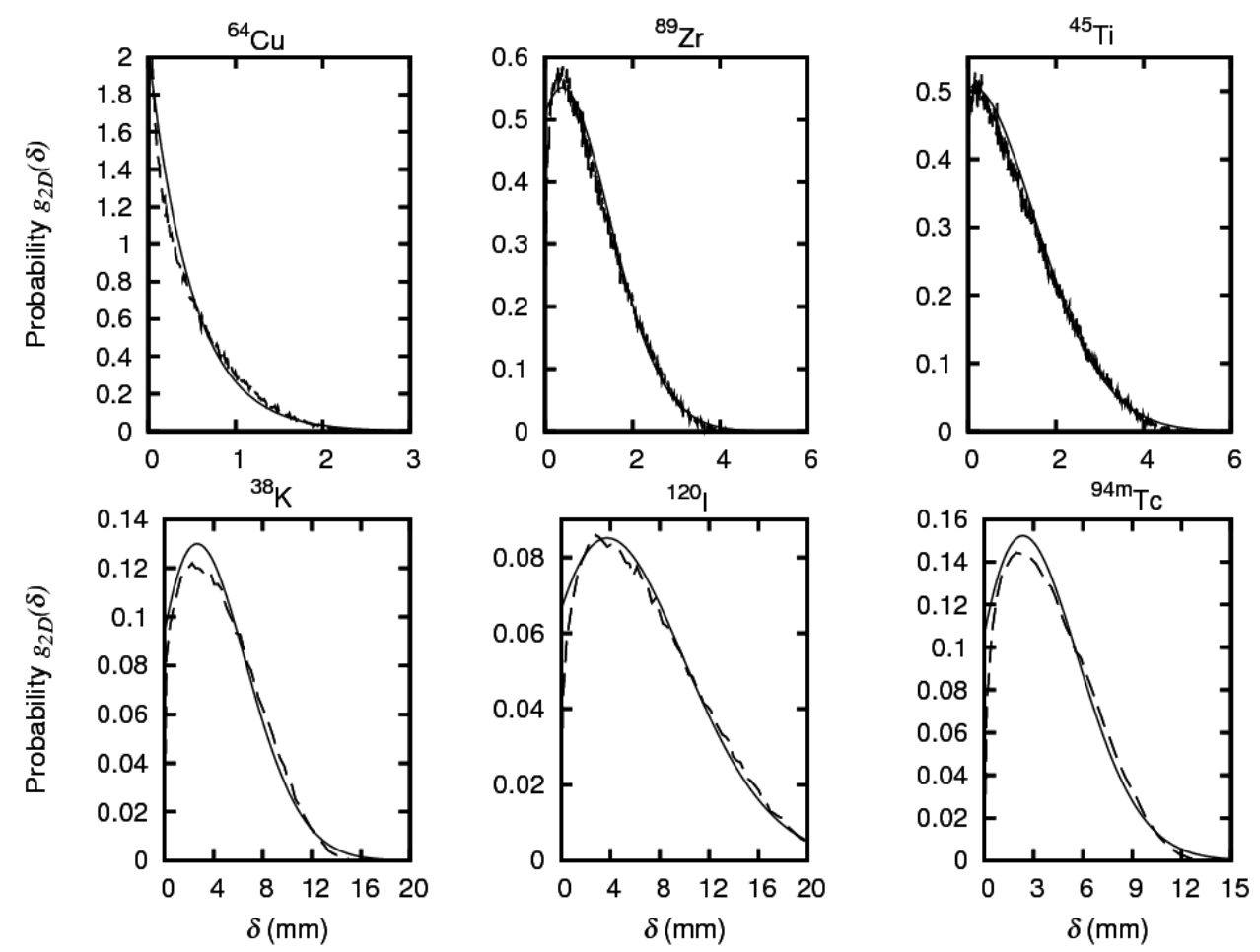

Figure 5: Functions $g_{2 D}(\delta)$ obtained from Monte Carlo calculations (broken lines) compared with the expressions defined by (3) using the values of $A$ and $B$ provided in table 2 (smooth lines).
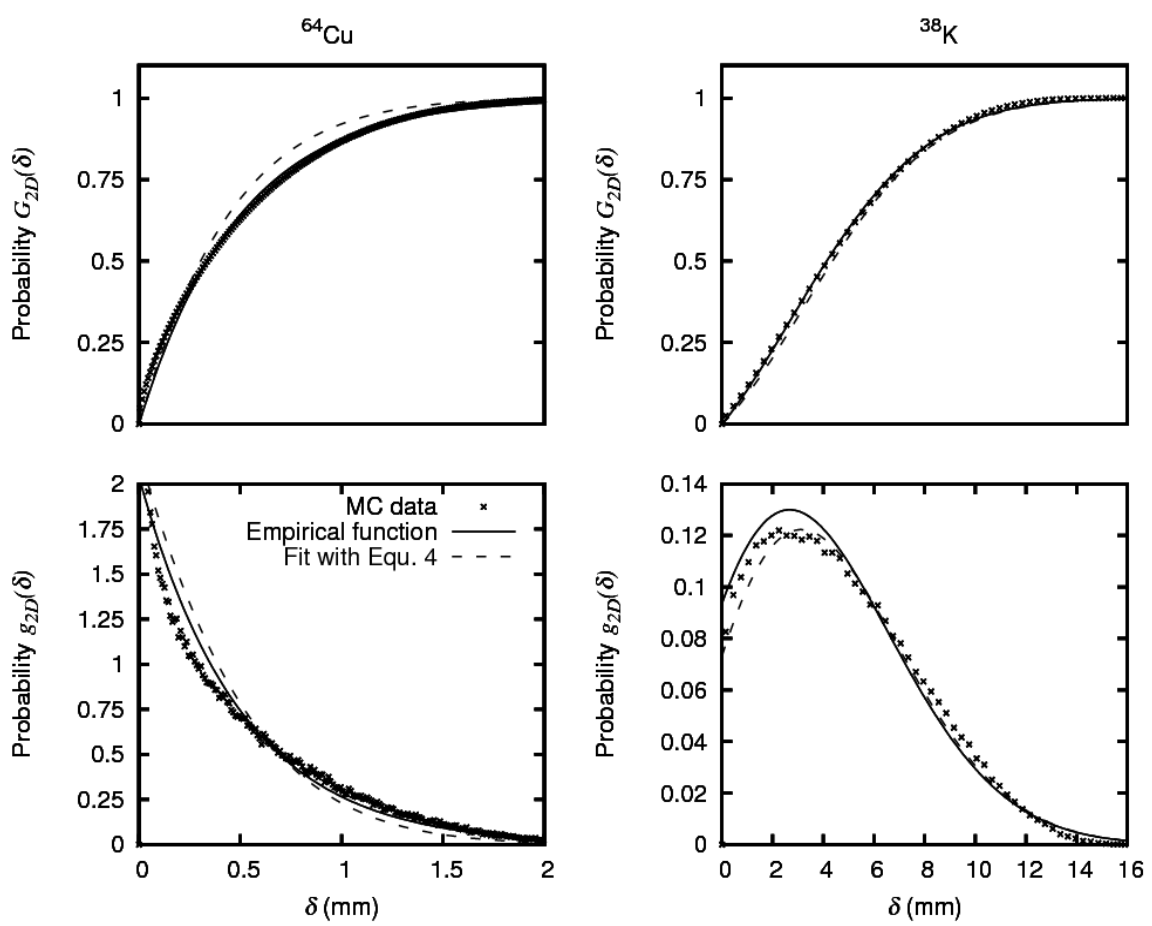
Figure 6: Functions $g_{2 D}(\delta)$ and $G_{2 D}(\delta)$ obtained from Monte Carlo calculations (crosses) compared with the expressions defined by (3) using the values of $A$ and $B$ provided in table 2 (smooth lines) and the expressions defined in (4) (broken lines).
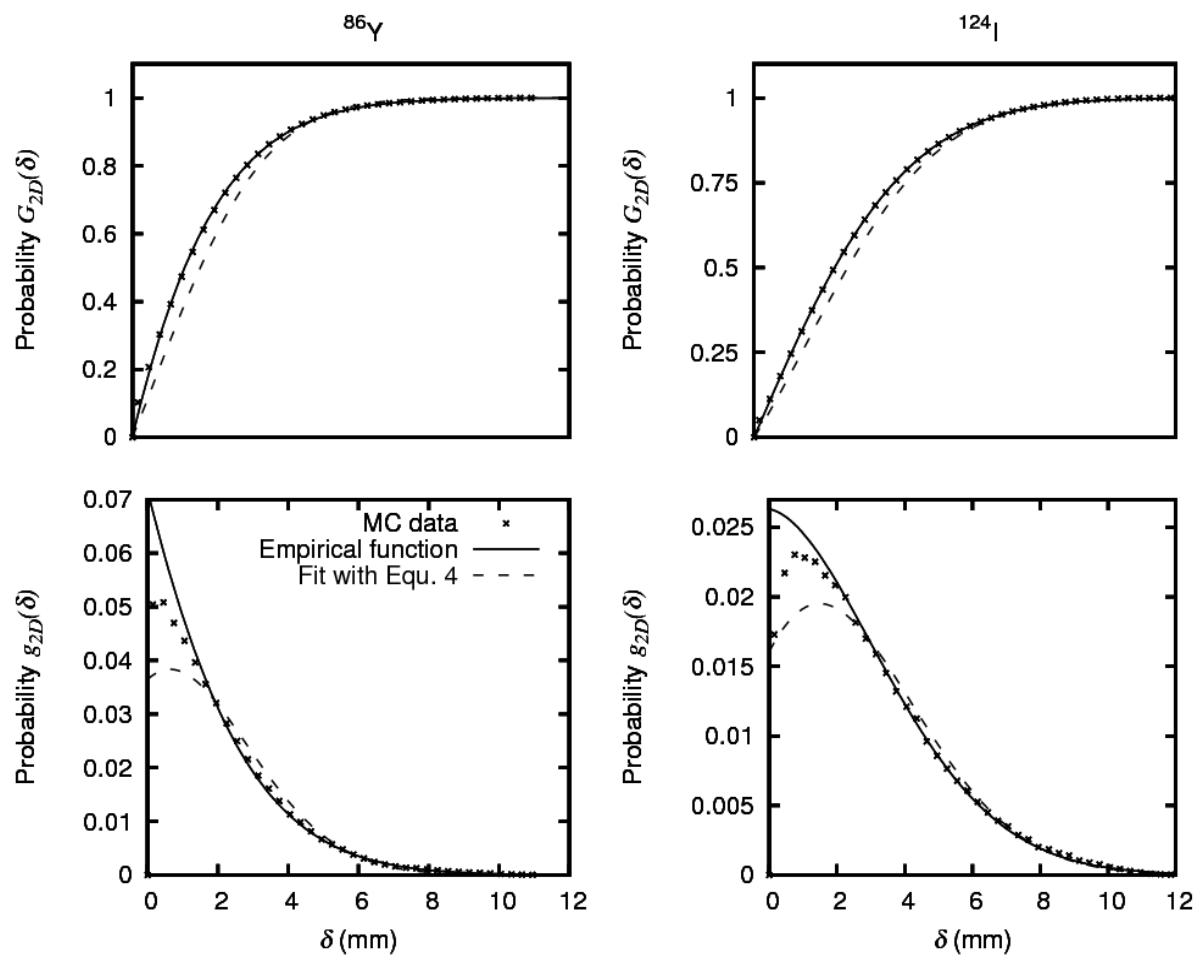

Figure 7: Same as figure 6 for the forbidden-decay isotopes ${ }^{86} \mathrm{Y}$ and ${ }^{124} \mathrm{I}$.

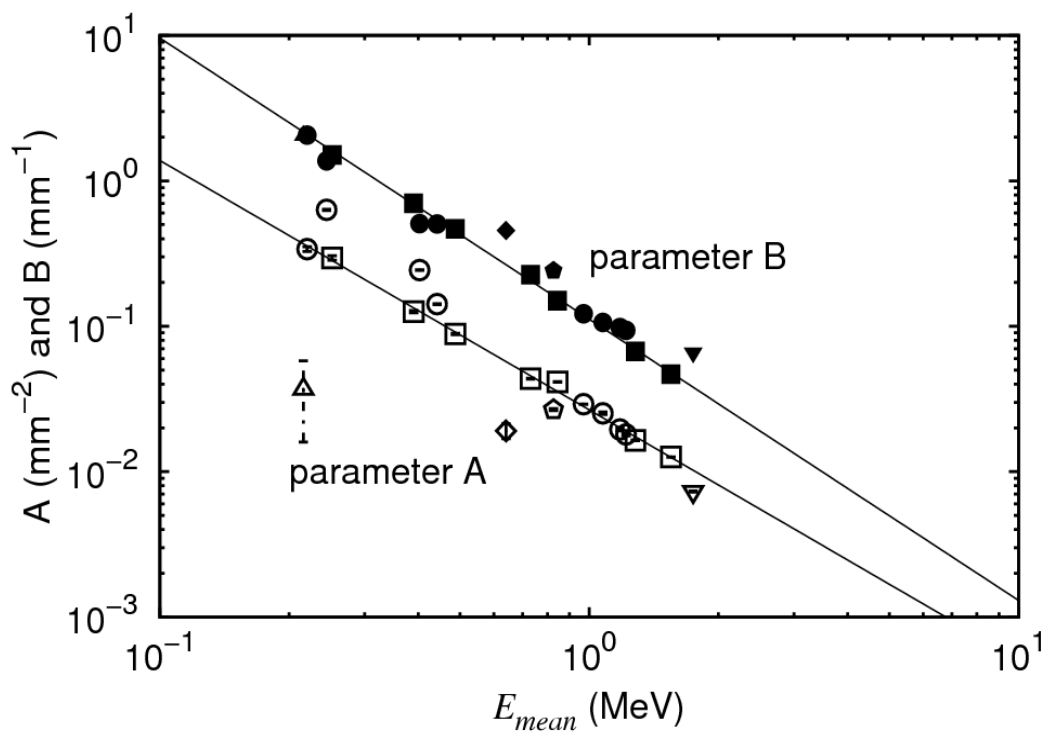


Figure 8: Parameters $A$ and $B$ plotted vs. $E_{\text {mean }}$ alongside the functions defined in Eq. (4). The isotopes that are represented include those investigated in the previous paper (squares), ${ }^{64} \mathrm{Cu}$ (upward triangles), ${ }^{120} \mathrm{I}$ (downward triangles), ${ }^{86} \mathrm{Y}$ (diamonds), ${ }^{124} \mathrm{I}$ (pentagons) and new allowed-decay isotopes (circles). The open symbols correspond to parameter $A$. The filled symbols correspond to parameter $B$.

\section{Comparison with the pattern observed for the previous results}

For the originally investigated allowed-decay isotopes, we empirically found that the best-fit values of $A$ and $B$ could be reasonably predicted by the formulae:

$$
A=0.0266\left(E_{\text {mean }}\right)^{-1.716} \text { and } B=0.1119\left(E_{\text {mean }}\right)^{-1.934}
$$

where the value of $E_{\text {mean }}$ must be in $\mathrm{MeV}$.

The results of the fits are reported in figure 8. To study the reliability of the method, we calculated, for each isotope, the reliability factor $R / R_{0}$, which is defined as follows:

$$
R / R_{0}=\sqrt{\frac{\sum\left(g_{2 D}^{M C}(\delta)-g_{2 D}^{E q 4}(\delta)\right)^{2}}{\sum\left(g_{2 D}^{M C}(\delta)-g_{2 D}^{e m p}(\delta)\right)^{2}}}
$$

where $g_{2 D}^{M C}(\delta)$ denotes the value of $g_{2 D}(\delta)$ determined from the Monte Carlo data, $g_{2 D}^{e m p}(\delta)$ is the value determined from the empirical functions (2)-(3) by the fit, and $g_{2 D}^{E q 4}(\delta)$ is the value found by calculating $A$ and $B$ by Eq. (4).

It is worth noting that this ratio is expected to be greater than 1 for all isotopes, as the empirical fit should theoretically provide a better description of the MC data than that provided by Eq. (4). Thus, for an ideal interpolation of $\mathrm{A}$ and $\mathrm{B}$, the ratio should be equal to 1 . The results are reported in figure 9. We note that 3 isotopes $\left({ }^{94 \mathrm{~m}} \mathrm{Tc},{ }^{52 \mathrm{~m}} \mathrm{Mn}\right.$ and $\left.{ }^{38} \mathrm{~K}\right)$ exhibit values of $R / R_{0}$ that are actually smaller than 1. For these 3 isotopes, the empirical fit well describes the first part of the $g_{2 D}$ distribution, but Eq. 
(4) provides a better description for the largest distances. Moreover, Eq. (4) seems to yield a better evaluation of parameter B overall than does the direct fit.

In accordance with the uncertainties associated with the MC data and the fitting procedure, we estimated that a reliability factor below 1.2 (i.e. at most $20 \%$ worse than the direct fit) would be acceptable for our study. Five of the isotopes studied here lie outside of these tolerance limits: ${ }^{120} \mathrm{I}$, ${ }^{124} \mathrm{I},{ }^{89} \mathrm{Zr},{ }^{52} \mathrm{Mn}$, and ${ }^{64} \mathrm{Cu}$. However, the resulting density functions presented in figures 4-7 display good agreement. For these isotopes, Eq. (4) is thus less successful than the direct fit, but it is still somewhat representative of the displacement probability spectrum.

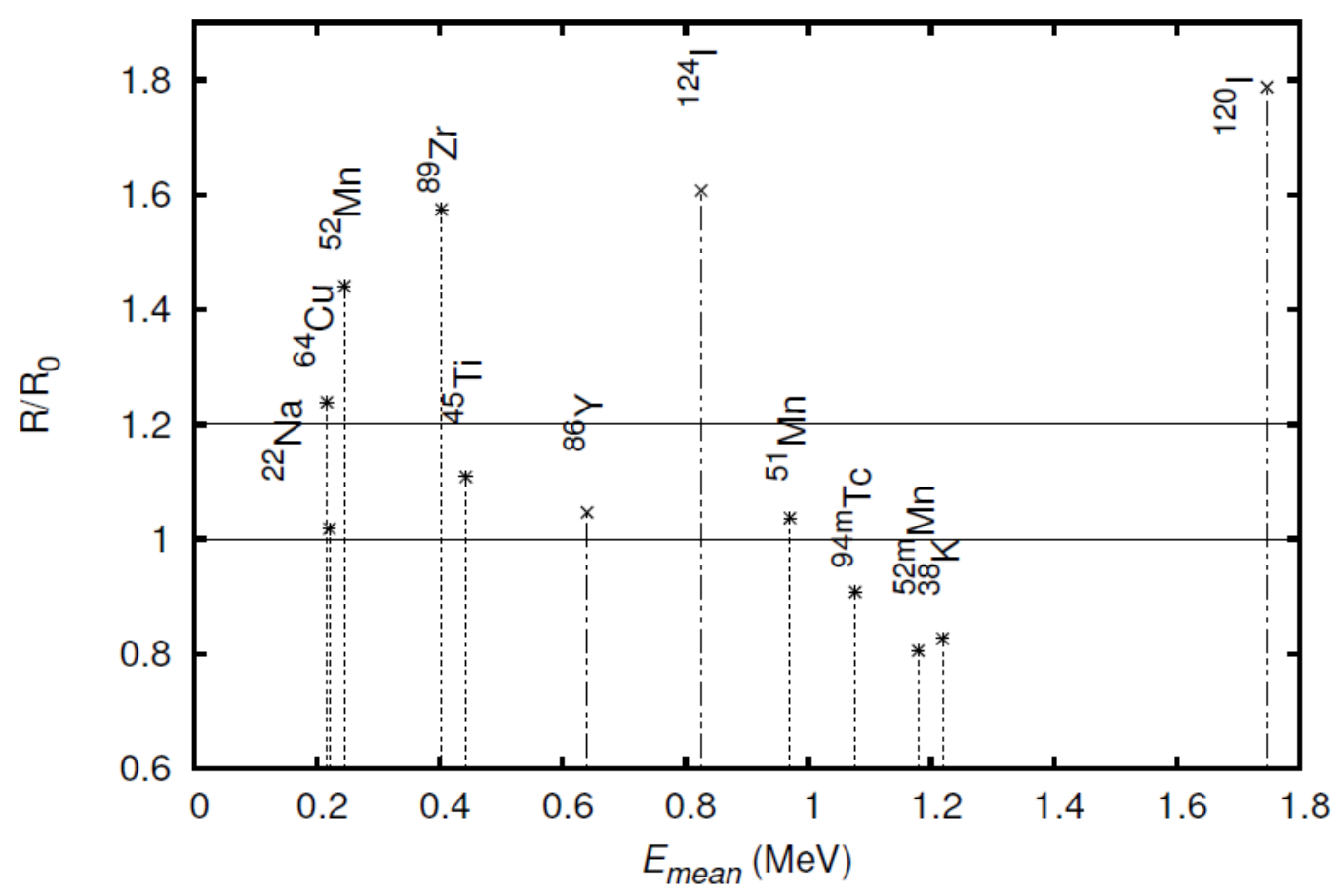

Figure 9: Reliability factors $R / R_{0}$ obtained for the various isotopes studied here (stars and dotted lines represent allowed isotopes crosses and discontinued lines represent forbidden isotopes).

\section{General discussion}


When PET isotopes emit their positrons with high energy, blurring associated with the positron range can be the limiting factor for spatial resolution. This is a particularly critical problem in micro PET, in which the intrinsic resolution of the scanner can be in the sub-millimeter range. If, however, the positron range is accounted for in the model used by the image-reconstruction software, the resolution can be improved.

Of course, the positron range is not the only source of resolution degradation. Other relevant factors include photon non-collinearity and the properties of the detection system. In recent years, PET scanners with "resolution-recovery" algorithms have become available. These algorithms usually account for all resolution-degrading effects and are typically based on measurements of pointlike sources for the determination of point-spread functions (Mourik et al. 2010; Narayanan and Perkins 2013; Wallsten et al. 2013). This is a simple and effective approach when only one isotope is to be used (e.g., ${ }^{18} \mathrm{~F}$ ) or when the contribution of the positron range is small compared to the other effects.

However, a point-spread function based on measurements with a single isotope will not correctly represent the spread from an isotope with a considerably different mean energy (having a considerably different positron range spectrum). If an improper point-spread function is used, the resolution-recovery algorithms may introduce artifacts because of improper correction.

An algorithm in which the point-spread function is adjusted depending on the actual isotope used would ameliorate this difficulty. It is possible to implement such an algorithm if the point-spread function can be modeled mathematically in such a manner that the influence of the positron range can be separated from that of the scanner-dependent properties. Such a model may be achieved by creating a full mathematical model of the scanner, or the scanner-part of the model may be determined from measured point-spread functions through deconvolution with a mathematical description of the positron range. 
As discussed by Cal-González (2013), positron-range distributions can be represented in many forms, all of which represent the same distribution but for which conversions between forms are nontrivial. We chose a 2-dimensional representation because this representation corresponds to the information that the detectors actually measure (cf. Figure 1), and we represented the distributions as radial distributions because doing so avoids the very peaked (cusp-shaped) distributions that are obtained when non-radial distributions are considered.

The relatively simple, empirical models for the radial cumulative distribution $G_{2 \mathrm{D}}(\delta)$, as described by Eq. (2), and the radial density distribution $g_{2 \mathrm{D}}(\delta)$, as described by Eq. (3), proved to perform well for most of the isotopes studied here, even confirming the validity of Eq. (4) for parameters $A$ and $B$.

For 4 isotopes, the estimation formulae of Eq. (4) for parameters $A$ and $B$ tended to be less accurate but nevertheless yielded a reasonable approximation of the displacement probability function.

\section{Conclusion}

We extended our previous work concerning the positron-range distributions of conventionally used PET isotopes to a number of non-conventional isotopes. The results for the allowed-decay isotopes indicated that comparable performance was achieved using the same approach developed for the conventional (also allowed-decay) isotopes, except in the case of ${ }^{64} \mathrm{Cu}$, for which the values of the fitting parameters differed. These findings are intended for use in reconstruction algorithms that account for an isotope-dependent positron range.

\section{References}


Alessio A and MacDonald, L. 2008 Spatially variant positron range modeling derived from CT for PET image image reconstruction. IEEE Nucl. Sci. Symp. Conf. Rec. 3637-3640.

Bai B, Ruangma, A, Laforest, R, Tay, YC, and Leahy, RM. 2003 Positron range modeling for statistical PET image reconstruction. IEEE Nucl. Sci. Symp. Conf. Rec. 4 2501-2505.

Bol A, Baudhuin, T, DePauw, M, Cogneau, M, Labar, D, Vanbutsele, R, Heyndrickx, G, Michel, C, Wijns, W, and Melin, JA. 1993 Quantification of absolute myocardial perfusion with K-38 and positronemission tomography. J. Nucl. Med. $34 \mathrm{P} 86$.

Cal-Gonzalez J, Herraiz, JL, Espana, S, Corzo, PM, Vaquero, JJ, Desco, M, and Udias, JM. 2013 Positron range estimations with PeneloPET. Phys. Med. Biol. 58 5127-5152.

Champion C and Le Loirec, C. 2007 Positron follow-up in liquid water: II. Spatial and energetic study for the most important radioisotopes used in PET. Phys. Med. Biol. 52 6605-6625.

Champion C, Le Loirec, C, and Stosic, B. 2012 EPOTRAN: a full-differential Monte Carlo code for electron and positron transport in liquid and gaseous water. Int. J. Radiat. Biol. 88 54-61.

Daghighian F, Pentlow, KS, Larson, SM, Graham, MC, DiResta, GR, Yeh, SD, Macapinlac, H, Finn, RD, Arbit, E, and Cheung, NK. 1993 Development of a method to measure kinetics of radiolabelled monoclonal antibody in human tumour with applications to microdosimetry: positron emission tomography studies of iodine-124 labelled 3F8 monoclonal antibody in glioma. Eur. $J$. Nucl. Med. 20 402-409.

Daube ME and Nickles, RJ. 1985 Development of myocardial perfusion tracers for positron emission tomography. Int. J. Nucl. Med. Biol. 12 303-314.

de Jong HWAM, Perk, L, Visser, GWM, Boellard, R, van Dongen, GAMS, and Lammertsma, AA. 2005 High resolution PET imaging characteristics of Ga-68, I-124 and Zr-89 compared to F-18. IEEE Nucl. Sci. Symp. Conf. Rec. 1627.

De Landsheere C, Mannheimer, C, Habets, A, Guillaume, M, Bourgeois, I, Augustinsson, LE, Eliasson, T, Lamotte, D, Kulbertus, H, and Rigo, P. 1992 Effect of spinal cord stimulation on regional myocardial perfusion assessed by positron emission tomography. Am. J. Cardiol. $691143-$ 1149.

Disselhorst JA, Brom, M, Laverman, P, Slump, CH, Boerman, OC, Oyen, WJ, Gotthardt, M, and Visser, EP. 2010 Image-quality assessment for several positron emitters using the NEMA NU 42008 standards in the Siemens Inveon small-animal PET scanner. J. Nucl. Med. 51 610-617.

Herzog H, Qaim, SM, Tellmann, L, Spellerberg, S, Kruecker, D, and Coenen, HH. 2006 Assessment of the short-lived non-pure positron-emitting nuclide ${ }^{120} \mathrm{I}$ for PET imaging. Eur. J. Nucl. Med. Mol. Imaging 33 1249-1257.

Herzog H, Rosch, F, Stocklin, G, Lueders, C, Qaim, SM, and Feinendegen, LE. 1993 Measurement of pharmacokinetics of yttrium-86 radiopharmaceuticals with PET and radiation dose calculation of analogous yttrium-90 radiotherapeutics. J. Nucl. Med. 34 2222-2226. 
Hui JCK, Atkins, HL, Som, P, Ku, TH, Fairchild, RG, Giwa, LO, and Richards, P. 1979 Manganese- $52 \mathrm{~m}$ positron emission transaxial tomography for detecting myocardial infarction. $J$. Nucl. Med. 20648.

Jødal L, Le Loirec, C, and Champion, C. 2012 Positron range in PET imaging: an alternative approach for assessing and correcting the blurring. Phys. Med. Biol. 57 3931-3943.

Kemerink GJ, Visser, MG, Franssen, R, Beijer, E, Zamburlini, M, Halders, SG, Brans, B, Mottaghy, FM, and Teule, GJ. 2011 Effect of the positron range of ${ }^{18} \mathrm{~F},{ }^{68} \mathrm{Ga}$ and ${ }^{124} \mathrm{I}$ on PET/CT in lung-equivalent materials. Eur. J. Nucl. Med. Mol. Imaging 38 940-948.

Krane, KS. 1988 Beta decay, in Introductory Nuclear Physics, 2nd ed, (John Wiley \& Sons, pp. 289-291).

Laforest R, Rowland, DJ, and Welch, MJ. 2002 MicroPET imaging with nonconventional isotopes. IEEE Trans. Nucl. Sci. 49 2119-2126.

Larson SM, Pentlow, KS, Volkow, ND, Wolf, AP, Finn, RD, Lambrecht, RM, Graham, MC, Di Resta, G, Bendriem, B, Daghighian, F, Yeh, SDJ, Wang, GJ, and Cheung, NKV. 1992 PET scanning of iodine-124-3F9 as an approach to tumor dosimetry during treatment planning for radioimmunotherapy in a child with neuroblastoma. J. Nucl. Med. 33 2020-2023.

Le Loirec C. 2007 Simulation Monte Carlo de suivi de positrons dans la matière biologique: Applications en imagerie médicale. PhD Thesis .

Le Loirec C and Champion, C. 2007a Track structure simulation for positron emitters of physical interest. Part II: The case of the radiometals. Nucl. Instrum. Methods Phys.Res., Sect.A $582654-$ 664.

Le Loirec C and Champion, C. 2007b Track structure simulation for positron emitters of physical interest. Part III: The case of the non-standard radionuclides. Nucl. Instrum. Methods Phys. Res., Sect.A 582 665-672.

Lederer, CM, Hollander, JM, \& Perlman, I 1967, Table of Isotopes, 6th edn, John Wiley \& Sons.

Link JM, Krohn, KA, Eary, JF, Kishore, R, Lewellen, TK, Johnson, MW, Badger, CC, Richter, KY, and Nelp, WB. $2006 \mathrm{Zr}-89$ for antibody labeling and positron emission tomography. J. Label. Comp. Radiopharm. 23 1297-1298.

Liu X and Laforest, R. 2007 Ga-68, Tc-94m, Y-86, and Cu-61 imaging performance in microPET. J. Nucl. Med. 48(Suppl 2) 412P.

Liu X and Laforest, R. 2009 Quantitative small animal PET imaging with nonconventional nuclides. Nucl. Med. Biol. 36 551-559.

Mourik JE, Lubberink, M, van Velden, FH, Kloet, RW, van Berckel, BN, Lammertsma, AA, and Boellaard, R. 2010 In vivo validation of reconstruction-based resolution recovery for human brain studies. J. Cereb. Blood Flow Metab. 30 381-389. 
Narayanan, M. and Perkins, A. 16-12-2013 Resolution recovery in the Ingenuity TF PET/CT. Available from: URL: http://clinical.netforum.healthcare.philips.com/global/Explore/WhitePapers/CT/Resolution-recovery-in-the-Ingenuity-TF-PET-CT

Pagani M, Stone-Elander, S, and Larsson, SA. 1997 Alternative positron emission tomography with non-conventional positron emitters: effects of their physical properties on image quality and potential clinical applications. Eur. J. Nucl. Med. 24 1301-1327.

Philpott GW, Schwartz, SW, Anderson, CJ, Dehdashti, F, Connett, JM, Zinn, KR, Meares, CF, Cutler, PD, Welch, MJ, and Siegel, BA. 1995 RadioimmunoPET: detection of colorectal carcinoma with positron-emitting copper-68-labeled monocolonal antibody. J. Nucl. Med. 36 1818-1824.

Rösch F, Herzog, H, Plag, C, Neumaier, B, Braun, U, Müller-Gärtner, HW, and Stöcklin, G. 1996 Radiation doses of yttrium-90 citrate and yttrium-90 EDTMP as determined via analogous yttrium86 complexes and positron emission tomography. Eur. J. Nucl. Med. 23 958-966.

Sastri CS, Petri, H, Kueppers, G, and Erdtmann, G. 1981 Production of manganese-52 of high isotropic purity by ${ }^{3} \mathrm{He}$-activation of vanadium. Int. J. Appl. Radiat. Isotopes 32 246-247.

Snyder, WS, Ford, MR, Warner, GG, \& Warson, SB 1975, MIRD Pamphlet No. 11 New York: Society of Nuclear Medicine.

Tolmachev, V, Bruskin, A, and Lunqvist, H. 1994 Preliminary study of production routes of positron emitting isotopes of manganese, in Neutron deficient nuclides for positron emission tomography, (Moscow: Institute of Theoretical and Experimental Physics, pp. 47-94).

Topping G J, Schaffer P, Hoehr C, Ruth T J and Sossi V 2013 Manganese-52 positron emission tomography tracer characterization and initial results in phantoms and in vivo. Med. Phys. 40 042502

Vavere AL and Welch, MJ. 2005 Preparation, biodistribution, and small animal PET of ${ }^{45} \mathrm{Ti}-$ transferrin. J. Nucl. Med. 46 683-690.

Venkataramaiah P, Gopala, K, Basavaraju, A, Suryanarayana, SS, and Sanjeeviah, H. 1985 A Simple Relation for the Fermi Function. J. Phys. G: Nucl. Part. Phys. 11 359-364.

Wallsten E, Axelsson, J, Sundstrom, T, Riklund, K, and Larsson, A. 2013 Subcentimeter tumor lesion delineation for high-resolution 18F-FDG PET images: optimizing correction for partialvolume effects. J. Nucl. Med. Technol. 41 85-91.

Zweit J, Flower, M, Brown, A, Carnochan, P, Luthra, S, Brady, F, Pike, V, Vaidyanathan, G, Zalutsky, M, Ott, R, and Jones, T. 1996 Iodine-120-mIBG: Production and NCA labelling of a new PET radiotracer. J. Nucl. Med. 37(Suppl) 874.

Derenzo SE. Mathematical removal of positron range blurring in high-resolution tomography. IEEE Trans Nucl Sci. 1986;NS-33:565-569.

Levin CS and Hoffman EJ. Calculation of positron range and its effect on the fundamental limit of positron emission tomography system spatial resolution. Phys Med Biol. 1999;44:781-99. 